\title{
Clinical experience treating Paecilomyces lilacinus keratitis in four patients
}

This article was published in the following Dove Press journal:

Clinical Ophthalmology

21 June 2012

Number of times this article has been viewed

Yu Monden'

Minoru Sugita'

Ryoji Yamakawa'

Kazuko Nishimura ${ }^{2}$

'Department of Ophthalmology, Kurume University School of Medicine, Kurume, Fukuoka, Japan; ${ }^{2}$ Medical Mycology Research Center, Chiba University and First Laboratories Co Ltd, Kawasaki, Kanagawa, Japan
Correspondence: Yu Monden Department of Ophthalmology, Kurume University School of Medicine, 67 Asahi-machi, Kurume 830-00I I, Japan Tel $+8 \mid 942317574$

Fax+8I 942370324

Email you@med.kurume-u.ac.jp
Background: Paecilomyces lilacinus is a saprobic fungus that occasionally causes keratitis in infected patients. Voriconazole, a triazole antifungal agent, is often administered to treat P. lilacinus keratitis, because it is resistant to many antifungal agents. However, some patients may not require voriconazole. Here, we report our experience of treating this infection and compare outcomes between patients treated with or without voriconazole.

Subjects: We retrospectively reviewed four cases of infectious keratitis caused by P. lilacinus and compared treatment course and outcomes among the four cases.

Observations: P. lilacinus was isolated from corneal cultures in all four cases. Three cases developed corneal perforation and underwent keratoplasty. Voriconazole was given in two cases with severe and refractory infection. Both required long-term treatment despite the effectiveness of voriconazole. They also had a medical history of diabetes and corticosteroid therapy. In two cases that were not treated with voriconazole, the eye conditions improved with a short treatment period (2-3 weeks). Neither of these cases had a medical history of diabetes, nor had they used corticosteroids.

Conclusion: Although voriconazole is the most useful antifungal agent for treating P. lilacinus keratitis, this infection can be resolved by other treatments. Voriconazole should be offered to patients with diabetes and/or prior corticosteroid use.

Keywords: Paecilomyces lilacinus, voriconazole, diabetes, corticosteroids

\section{Introduction}

Paecilomyces lilacinus is a filamentous fungus found in air and soil, which sometimes causes ocular infection. Although P. lilacinus keratitis is resistant to many antifungal agents, voriconazole has been established as an effective treatment for this disease. ${ }^{1-4}$ However, it seems likely that the increasing use of voriconazole may lead to the emergence of strains resistant to this drug. Therefore, it is important to consider whether the use of voriconazole is essential or whether other treatments could be used instead.

To inform on this clinical setting, we performed a retrospective review of four patients with P. lilacinus keratitis who were treated at our hospital. Two patients were treated with voriconazole and two without voriconazole. We compared the clinical courses, treatment approaches, and outcomes among the four patients. Our aim was to determine whether voriconazole is essential to treat $P$. lilacinus keratitis, and hence provide guidance on how best to treat this infection. 


\section{Methods}

We performed a retrospective review of cases of $P$. lilacinus keratitis treated at our hospital. We extracted information from their medical records, including clinical presentation, treatment course, and outcomes. This study was approved by the Ethical Committee of Kurume University, Fukuoka, Japan, who waived the need for informed consent to publish the details of these patients.

\section{Results}

\section{Case I}

A 57-year-old man with a history of diabetes experienced trauma to his left eye while cutting grass on 19 November 2005. Despite receiving antibiotics and corticosteroids from his local ophthalmologist, his eye condition worsened. He was referred to our hospital on January 24, 2006.

At presentation, he complained of severe pain, redness, and decreasing vision in the left eye. He had a history of laser photocoagulation for retinal hemorrhage in the left eye before attending our hospital. Visual acuity (VA) in the left eye was limited to hand motion. Slit-lamp examination showed a round central corneal infiltrate with feathery margins (Figure 1A). Microscopic examination of corneal scrapings revealed a budding yeast-like organism, and an initial diagnosis of yeast keratitis was made. The patient was admitted into the hospital on the same day as the presentation, and the corticosteroids were discontinued. Treatment consisting of topical $0.1 \%$ fluconazole, $0.1 \%$ miconazole every hour, and intravenous fluconazole $800 \mathrm{mg}$ a day was initiated. Corneal debridement was performed every second day.

One week later, the corneal infiltrate increased, and satellite lesions and an immune ring were found. A filamentous fungus was therefore suspected of being the cause of the infection. As a result, fluconazole was discontinued and the patient was switched to intravenous miconazole. Ophthalmic 1\% pimaricin ointment was administered six times per day. Corneal debridement was performed every day. Four days later, the infiltrate further worsened and hypopyon appeared. Miconazole was therefore changed to voriconazole.

One week later, after starting voriconazole, the infiltrate worsened (Figure 1B), and P. lilacinus was isolated from the corneal culture (Figure 1C and D). Therefore, the patient was diagnosed with filamentous fungal keratitis. The dose of intravenous voriconazole was increased to $4 \mathrm{mg} / \mathrm{kg}$ twice a day, and topical $1 \%$ voriconazole every hour was changed to half-hourly. One week later, the infiltrate diminished (Figure 1E).
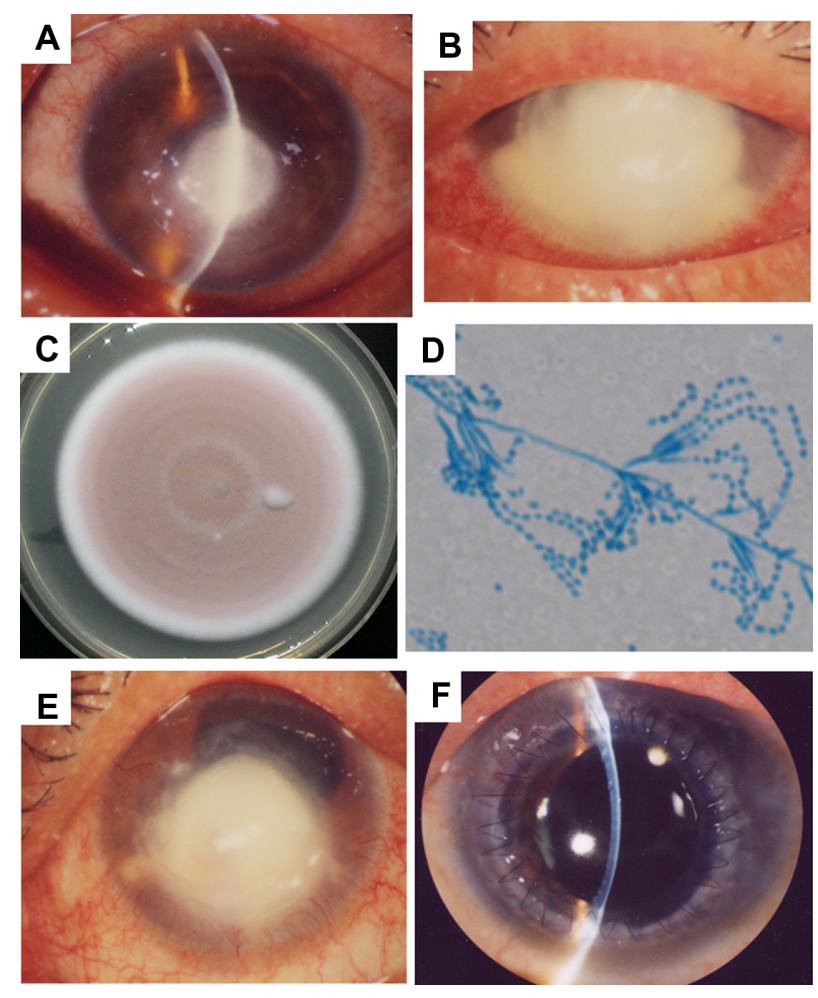

Figure I Case I. (A) The round, central corneal infiltrate with feathery margins is surrounded by a severely edematous cornea. (B) Enlargement of the infiltrate at 2.5 weeks after discontinuing corticosteroids and starting antifungal agents. (C) Colony of Paecilomyces lilacinus isolated from cornea, and the color of colony in 14 days is lilac on potato dextrose agar. (D) Photomicrograph of $P$. lilacinus demonstrating the long conidiophores arising from hyphae, tapering phialides, and chains of conidia (lactophenol cotton blue stain). (E) Two weeks after starting treatment with voriconazole, the infiltrate has decreased in size. (F) Two years after the discharge, the cornea is clear and no recurrence of fungal infection has been reported.

One week later, the center of the infiltrate became thinner and was at risk of corneal perforation. Therapeutic penetrating keratoplasty was therefore performed, with a good postoperative course. He was discharged from hospital on April 21, 2006, 3 months after admission. His VA at the last visit was 0.1 (Figure 1F). No recurrence of the fungal infection has been reported to date.

\section{Case 2}

A 64-year-old male farmer with history of diabetes developed hyperemia in the left eye in July 2006. He was diagnosed with scleritis by his local ophthalmologist and treated with corticosteroid eyedrops and subconjunctival injection. However, his eye condition worsened, and he was referred to our hospital on October 30, 2006.

His VA on presentation was 0.04 in the left eye. Slit-lamp examination showed nasal scleral hyperemia and a nasal peripheral corneal infiltrate with feathery margins (Figure 2A). Therefore, infectious keratitis was suspected, and corticosteroids were switched to antibiotics. The day 



Figure 2 Case 2. (A) Nasal scleral hyperemia and nasal peripheral corneal infiltrate with feathery margins. (B) Enlargement of the corneal infiltrate 2 days after discontinuing treatment with corticosteroids and starting antifungal agents. (C) Even though voriconazole decreased the size of the corneal infiltrate, the peripheral cornea is perforated. A fibrous clot was found in the anterior chamber. (D) One year after the discharge, the cornea is clear, and no recurrence of fungal infection has been reported.

after presentation, the corneal infiltrate worsened, and the patient was admitted to the hospital. No organism was found on smears of the original corneal scrapings.

Three days after admission, the corneal infiltrate increased with satellite lesions, an immune ring, and an endothelial plaque. Because fungal keratitis was suspected, his treatment was changed to topical $0.1 \%$ fluconazole, $0.1 \%$ miconazole, and intravenous fluconazole $800 \mathrm{mg} \mathrm{a}$ day. Two days later, hypopyon appeared (Figure 2B), and P. lilacinus was isolated from the corneal culture. A drug susceptibility test indicated susceptibility to voriconazole, and the treatment was changed to topical voriconazole $1 \%$ and intravenous voriconazole $3 \mathrm{mg} / \mathrm{kg}$ twice a day. Two weeks later, the infiltrate had improved, but the cornea was perforated (Figure 2C). Therefore, the patient underwent lamellar keratoplasty.

The patient's postoperative course was good, and he was discharged from the hospital on December 19, 2006. His VA at the last visit was 0.2 (Figure 2D). No recurrence of the fungal infection has been reported to date.

\section{Case 3}

An 83-year-old female gardener experienced a foreign-body sensation in her right eye on October 23, 2007. She was diagnosed with infectious keratitis by her local ophthalmologist and treated with antibiotics. However, her eye condition remained unchanged, and she was referred to our hospital on November 12, 2007.
On presentation, she reported no history of ocular and systemic diseases. Her VA was 0.2 in the right eye. Slit-lamp examination showed a temporal peripheral corneal infiltrate with central thinning. Microscopic examination of smears from corneal scrapings revealed a yeast-like organism, suspected of being pseudohyphae, which prompted the diagnosis of fungal keratitis. The patient was admitted into the hospital on the same day as the presentation and treated with topical and intravenous fluconazole. The next day, the cornea was perforated (Figure 3A), and lamellar keratoplasty was performed. P. lilacinus was isolated from the corneal culture after the surgery.

The patient's postoperative course was good, and she was discharged from the hospital on December 5, 2007. Her VA at the last visit was 0.8 (Figure 3B). No recurrence of the fungal infection has been reported to date.

\section{Case 4}

An 84-year-old man experienced trauma to his left eye after hitting a tree branch in June 2010. He was diagnosed with infectious keratitis by his local ophthalmologist and treated with antibiotics. However, his eye condition remained unchanged, and he was referred to our hospital on June 11, 2010.

On presentation, he reported no history of ocular or systemic diseases. His VA was 0.9 in the left eye. Slit-lamp examination showed an upper peripheral corneal infiltrate with feathery margins (Figure 4A). Microscopic examination of corneal scrapings revealed filamentous fungi. Therefore, the patient was treated with ophthalmic $1 \%$ pimaricin ointment six times per day. P. lilacinus was subsequently isolated from the corneal culture. At a follow-up visit 2 weeks after starting treatment, his eye condition showed marked improvements. His VA at the last visit was 1.0 (Figure 4B). No recurrence of the fungal infection has been reported to date.

\section{Discussion}

P. lilacinus is a rare but potentially serious cause of filamentous fungal keratitis. This fungus is found in air, soil,
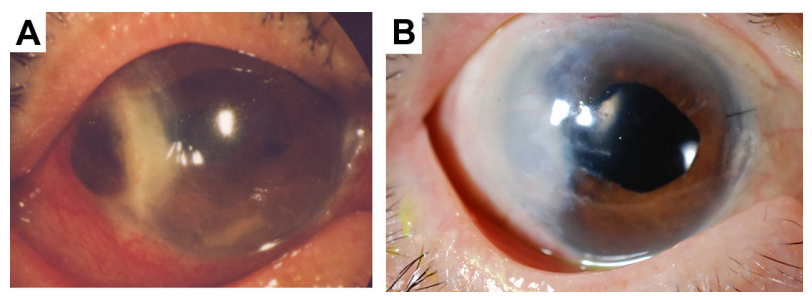

Figure 3 Case 3. (A) Perforation of the cornea I day after presentation at our hospital. (B) Eight months after surgery, the nasal cornea was clear, and the patient's visual acuity was 0.8 . 


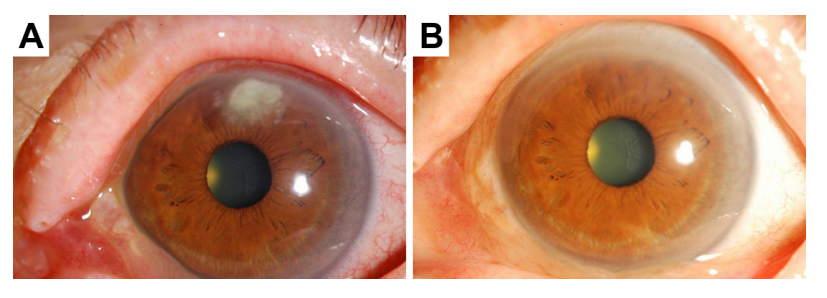

Figure 4 Case 4. (A) An upper peripheral corneal infiltrate with feathery margins. (B) Three months after starting treatment with antifungal agents, the cornea was clear, and the patient's visual acuity was I.0.

wood, and stored food, and mainly causes ocular infection in immunocompromised patients or following trauma. Microscopic examination of corneal scrapings revealed a yeast-like organism in cases 1 and 3, which prompted treatment for yeast infections. In another report, two cases were also misdiagnosed with yeast keratitis. ${ }^{5}$ It seems that $P$. lilacinus keratitis is commonly misdiagnosed as yeast keratitis based on initial microscopic findings, and hence patients are likely to be treated incorrectly. P. lilacinus grows quickly in the culture, and the color of colony is lilac. The microscopical features of P. lilacinus are long conidiophores arising from hyphae, tapering phialides, and chains of conidia. ${ }^{1}$ The microscopic findings of our cases were consistent with these features.

P. lilacinus keratitis is resistant to many antifungal agents, which means that empirical antifungal therapy may be ineffective in many cases. In cases 1 and 2, voriconazole was effective for the treatment of this eye infection, supporting prior reports. ${ }^{1-4}$ However, in reviewing the present cases, we noted that cases 1 and 2 were treated with voriconazole for up to 3 months. By contrast, the infiltrate resolved within 2-3 weeks of starting antifungal treatment in cases 3 and 4. Therefore, why was such a long treatment course necessary for cases 1 and 2 ?

We suspect that one reason for the longer treatment duration in cases 1 and 2 is that the diagnosis of fungal keratitis was delayed because corneal inflammation was suppressed by the early treatment with corticosteroids. As a result, despite treatment with antifungal agents, the corneal infiltrate and inflammation rapidly worsened in cases 1 and 2 after discontinuing corticosteroids (Figures $1 \mathrm{~B}$ and $2 \mathrm{~B}$ ). By contrast, fungal keratitis was diagnosed within 2-3 weeks in cases 3 and 4 . The corneal infiltrate and inflammation did not worsen in cases 3 and 4 after starting treatment with antifungal agents. Accordingly, we believe that the immunocompromised state in cases 1 and 2, as a result of corticosteroid use and history of diabetes, reduced the efficacy of the treatments applied in both cases. In addition, the delayed initiation of antifungal agents in these patients probably allowed the fungus to become more established and difficult to eradicate.

It is also important to note that keratoplasty was performed to treat corneal perforation in cases 1,2 , and 3 , as reported elsewhere..$^{2,3,5,7-9}$ Interestingly, cases 1 and 2 underwent surgical intervention after starting treatment with antifungal agents, whereas case 3 underwent surgical intervention on the day after the initial presentation. The postoperative courses were good in all three cases. However, considering our experience of case 4, prompt initiation of antifungal treatment may have avoided the need for surgery in these cases.

Here, we have reported our experience of four cases of P. lilacinus keratitis. All four patients were treated successfully, with good VA at discharge. Two of the cases ultimately received voriconazole, while the other two cases received other antifungal drugs. These positive outcomes raise the question of how best to treat eye infections, particularly considering the risk of blindness, and when/if voriconazole should be used. Based on our experience, we recommend that patients showing no improvement in infiltrate despite empirical therapy in combination with corticosteroids should promptly receive voriconazole. We also suggest that corneal scrapings should be obtained and cultured as early as possible to ensure that appropriate antibiotic or antifungal treatments are initiated in a timely manner. We believe that is important to establish treatment algorithms to help guide ophthalmologists in selecting and initiating the appropriate drug.

\section{Disclosure}

The authors have no conflict of interest to disclose.

\section{References}

1. Ford JG, Agee S, Greehaw ST. Successful medical treatment of a case of Paecilomyces lilacinus keratitis. Cornea. 2008;27(9):1077-1079.

2. Deng SX, Kamal KM, Hollander DA. The use of voriconazole in the management of post-penetrating keratoplasty Paecilomyces keratitis. J Ocul Pharmacol Ther. 2009;25(2):175-177.

3. Yuan X, Wilhelmus KR, Matoba AY, Alexandrakis G, Miller D, Huang AJW. Pathogenesis and outcome of Paecilomyces keratitis. Am J Ophthalmol. 2009;147(4):691-696.

4. Wu PC, Lai CH, Tan HY, Ma DHK, Hsiao CH. The successful medical treatment of a case of Paecilomyces lilacinus keratitis. Cornea. 2010;29(3):357-358.

5. Starr MB. Paecilomyces keratitis: two case reports in extended wear contact lens wearers. CLAO J. 1987;13(2):95-101.

6. Klont RR, Eggink CA, Rijs AJMM, Wesseling P, Verweij PE. Successful treatment of Fusarium keratitis with cornea transplantation and topical and systemic voriconazole. Clin Infect Dis. 2005;40(12): e110-e112.

7. Okhravi N, Dart JK, Towler HM, Lightman S. Paecilomyces lilacinus endophthalmitis with secondary keratitis. Arch Ophthalmol. 1997;115(10): $1320-1324$. 
8. Anderson KL, Mitra S, Salouti R, Pham TA, Taylor HR. Fungal keratitis caused by Paecilomyces lilacinus associated with a retained intracorneal hair. Cornea. 2004;23(5):516-521.
9. Malecha MA, Tarigopula S, Malecha MJ. Successful medical treatment of Paecilomyces lilacinus keratitis in a patient with a history of herpes simplex virus keratitis. Cornea. 2006;25(10):1240-1242.
Clinical Ophthalmology

\section{Publish your work in this journal}

Clinical Ophthalmology is an international, peer-reviewed journal covering all subspecialties within ophthalmology. Key topics include: Optometry; Visual science; Pharmacology and drug therapy in eye diseases; Basic Sciences; Primary and Secondary eye care; Patien Safety and Quality of Care Improvements. This journal is indexed on

Submit your manuscript here: http://www.dovepress.com/clinical-ophthalmology-journal

\section{Dovepress}

PubMed Central and CAS, and is the official journal of The Society of Clinical Ophthalmology (SCO). The manuscript management system is completely online and includes a very quick and fair peer-review system, which is all easy to use. Visit http://www.dovepress.com/ testimonials.php to read real quotes from published authors. 